\title{
The role of chemokine receptors in acute lung allograft rejection
}

\author{
S. Geleff*, D. Draganovici ${ }^{\#}$, P. Jaksch ${ }^{\top}$ and S. Segerer ${ }^{+, \$}$
}

ABSTRACT: Recruitment of inflammatory cells to vascularised allografts is a hallmark of rejection, and paves the way for chronic allograft injury. Chemokines play pivotal roles in the directed movement of leukocytes. Herein, we define the distribution of chemokine receptors for the most common cell types during human lung allograft rejection as a prerequisite for therapeutic interventions.

Immunohistochemistry was performed on lung allograft biopsies from $\mathbf{5 4}$ patients for the chemokine receptors CCR5, CXCR3 and CXCR1 and the Duffy antigen/receptor for chemokines (DARC).

Perivascular infiltrates in acute lung rejection are composed of subsets of mononuclear cells expressing the chemokine receptors CXCR1, CXCR3 and CCR5. DARC-positive small vessels and capillary vessels were associated with sites of inflammation and their number was increased during episodes of acute lung rejection. DARC expression correlated with an increase in interstitial CCR5-positive T-cells and CXCR1-positive leukocytes. Leucokytic infiltrates in bronchial/bronchiolar rejection express CXCR1 and CXCR3.

This is the first study that demonstrates an induction of the chemokine binding protein DARC at sites of acute human lung allograft rejection. Co-localisation with the chemokine receptors CXCR1 and CCR5 may indicate a role for DARC expression during leukocyte adhesion and interstitial infiltration.

KEYWORDS: Chemokine receptors, CXCR1, CXCR3, Duffy antigen/receptor for chemokines, lung allograft rejection

ung transplantation has become a routine treatment option for terminal lung failure [1]. Lung allograft rejection is a lifethreatening complication, and bronchiolitis obliterans is thought to be the manifestation of chronic lung allograft rejection [2]. The rejection of a lung allograft is characterised by the recruitment of inflammatory cells, predominantly T-cells, to particular compartments in the lung. Mechanisms involved in this recruitment are incompletely understood.

Chemokines are chemotactic cytokines that share a motif of conserved cysteine residues in their primary structures [3]. Chemokines function via seven transmembrane-spanning receptors, which signal upon binding of chemokines of one subgroup and are, therefore, labelled accordingly (CC chemokine receptors (CCRs) and chemokine CXC motif receptors (CXCRs), etc.). Chemokines play pivotal roles in the recruitment of inflammatory cells to sites of injury, including vascularised allografts $[4,5]$. The cell type recruited depends, in part, on the pattern of chemokine receptors expressed. CXCR1 binds the ligand
CXCL8/interleukin (IL)-8, which is a major chemoattractant for neutrophils [3]. CXCL8/IL-8 protein was increased in allograft biopsies after reperfusion and its level of expression correlated with lung function [6]. In a lung ischaemiareperfusion model the expression of CXC chemokines was associated with neutrophil recruitment [7]. Furthermore, CXC chemokines were increased in lavages from allograft recipients with reperfusion injury [7]. CXCR3 and CCR5 are two receptors that are predominantly expressed on activated, T-helper type 1 (Th1) cells. In a rat lung transplantation model of acute rejection, the increased expression of CXCL9/ Mig (monokine induced by gamma interferon) and CXCL10/inducible protein (IP)-10 (both ligands for CXCR3) was associated with the recruitment of CXCR3-positive cells to the allograft [8]. Inhibition of CXCL9/Mig reduced the recruitment of CXCR3-positive cells, resulting in lower rejection scores [8]. The chemokines CXCL9/Mig and CCL5/RANTES (ligand of CCR5) were found in alveolar lavages of lung allograft recipients with acute rejection $[9,10]$.
AFFILIATIONS

${ }^{*}$ Clinical Institute of Pathology, Medical University of Vienna, and

'Dept of Surgery, Medical University of Vienna, Vienna, Austria.

${ }^{*}$ Medizinische Poliklinik, University of Munich, Germany.

+Division of Nephrology, University Hospital Zurich, and s Institute of Anatomy, University of Zurich, Zurich, Switzerland.

CORRESPONDENCE

S. Geleff

Clinical Institute of Pathology

Medical University of Vienna

Währinger Gürtel 18-20

A-1090 Vienna

Austria

E-mail: silvana.geleff@

meduniwien.ac.at

Received:

March 152009

Accepted after revision:

June 282009

First published online:

July 162009 
The Duffy antigen/receptor for chemokines (DARC) is a member of a growing family of chemokine binding proteins that do not signal [11]. These proteins can modify chemokine functions via scavenging, uptake, degradation or transcellular transport [12]. DARC binds pro-inflammatory CC and CXC chemokines, and shares ligands with CCR5 and CXCR1. This protein was first described as a blood group antigen, but it is also expressed on endothelial cells $[13,14]$. DARC was found to be upregulated on small vessels during pneumonia and in various forms of renal allograft rejection [15]. Therefore, DARC might modulate the chemokine milieu in lung allografts during rejection, but also during infection.

Herein, we describe the expression of the chemokine receptors CCR5, CXCR3 and CXCR1 and the chemokine binding protein DARC on a large series of lung allograft biopsies with acute allograft rejection.

\section{MATERIAL AND METHODS}

\section{Study population}

Transbronchial lung allograft biopsies from 54 patients (one biopsy per patient) who underwent surveillance bronchoscopy for clinical suspicion of infection or acute rejection (early type) or in the setting of obliterative bronchiolitis to rule out acute rejection (late type) were included in the study. In the latter group, cases without acute rejection were not included. Serial sections were used for histological diagnosis and immunohistochemical analysis. In addition to standard stainings, immunohistochemistry of consecutive sections were performed for the cytokeratin marker CAM5-2 and with an antibody against CD3 (cluster of differentiation antigen 3). These two immunohistochemical stainings were used to semiquantitatively assess T-cell infiltration and to grade acute pulmonary rejection according to the revised working formulation of the Lung Rejection Study Group on behalf of the International Society for Heart and Lung Transplantation (ISHLT) [16]. Each biopsy was evaluated at low magnification for obvious mononuclear infiltrates. The minimal number of parenchymal venules/arterioles required to evaluate rejection was a median range of one to six vessels per transbronchial biopsy. Acute rejection is graded according to the nature and extent of perivascular leukocyte infiltration (adventitia of small/medium-sized venoles and/or arterioles). In minimal acute rejection (A1) the infiltrate consists of CD3positive predominantly small lymphocytes, in mild (A2) and moderate (A3) rejection the infiltrate consisted of more activated, larger lymphocytes together with macrophages, eosinophils and neutrophils. Extension of the infiltrate into adjacent alveolar walls defines A3 rejection. Coexistent airway inflammation manifesting as bronchiolar/bronchial lymphomononuclear infiltrates was graded as follows. B0: none; B1: minimal; B2: mild; B3 moderate; B4 severe; and BX: ungradable (due to sampling). The cohort of the present study included cases of rejection grades A0, A2 and A3, all of which are defined entities readily identifiable at low magnification in transbronchial biopsies. We excluded minimal rejection A1 and severe rejection A4; the former because it is usually not subject to mandatory therapeutic intervention in our centre, the latter because of its rare appearance. The study was performed according to the institutional ethical standards and in accordance with the declaration of Helsinki.

\section{Immunohistochemistry}

Immunohistochemistry was performed on formalin-fixed, paraffin-embedded lung biopsies as described previously [17]. In brief, dewaxed, rehydrated tissue sections were incubated in 3\% hydrogen peroxide (to neutralise endogenous peroxidases). Antigen retrieval was performed in an autoclave oven using Antigen Retrieval Solution (Vector, Burlingame, CA, USA). The Avidin/Biotin blocking Kit (Vector) was used to block endogenous biotin. Incubation with the primary antibody was followed by incubation with biotinylated secondary antibodies (Vector), and the $\mathrm{ABC}$ reagent (Vector). 3'3'Diaminobenzidine (Sigma, Taufkirchen, Germany) with metal enhancement (resulting in a black product) was used as a detection system. As negative controls the primary antibody was replaced by isotype-matched irrelevant mouse immunoglobulin $G$ on tissue sections from human tonsils. The monoclonal antibodies used were anti-CD3 antibody (Novocastra, Newcastle-Upon-Tyne, UK), anti-cytokeratins 7/8 (clone CAM 5-2; Becton Dickinson and Company, Franklin Lakes, NJ, USA), MC5 (gift from M. Mack; University of Regensburg, Regensburg, Germany) against CCR5, anti- CXCR3 (clone 1C6; Becton Dickinson and Company), anti-CXCR1 (Research and Diagnostic Systems, Inc., Minneapolis, MN, USA) and 2C3 against DARC (gift from Y. Collins; INSERM U665, Institut National de la Transfusion Sanguine, Paris, France).

\section{Quantification}

The number of positive cells in the perivascular area of small to medium-sized arteries and veins (between one and seven medium-sized blood vessels per biopsy) was expressed as a percentage of the overall perivascular infiltrates. Each specimen was also scored for the presence or absence of DARC immunostaining on alveolar capillaries $(0=$ absent, $1=$ focal, at least one area per biopsy, $2=$ more than two separate foci) on medium-sized vessels and semiquantitatively for the presence of interstitial CCR5- and CXCR1-positive cells as follows. No infiltrate: 0; mild: 1; moderate: 2; and severe: 3 . All scores are presented as mean \pm SEM. Means were compared with the nonparametric Kruskal-Wallis test. A p-value $<0.05$ was considered statistically significant.

\section{RESULTS}

\section{Demographics and clinicopathological data}

The study group comprised of 37 males and 17 females, ranging in age from 15 to $68 \mathrm{yrs}$ (median age at the time of lung transplantation was $49 \mathrm{yrs}$ ). The median time between transplantation and allograft biopsy was 309 days (range 81,520 days). All patients received basal immunosuppression consisting of cyclosporine, mycophenolate mofetil and steroids. We included 24 specimens with mild acute rejection (A2), seven with severe acute rejection (A3) and 23 biopsies with no evidence of acute rejection (A0). To keep a stringent separation of entities we chose biopsies with no evidence of chronic bronchiolar and/or chronic vascular rejection. Biopsies with minimal rejection (A1) were also not included.

Lymphocytic bronchitis/bronchiolitis recognised as a possible harbinger of bronchiolitis obliterans [16] could be assessed in 28 out of 37 biopsies and graded B1-B3 (tables 1 and 2). The presence of concomitant acute and/or chronic infection was 
TABLE 1 Quantification of the immunohistochemical evaluation

\begin{tabular}{|c|c|c|c|c|c|c|c|c|}
\hline \multirow[t]{2}{*}{ Rejection } & \multicolumn{3}{|c|}{ Fraction of positive cells } & \multicolumn{5}{|c|}{ Score } \\
\hline & $\begin{array}{c}\text { CXCR3 } \\
\text { perivascular }\end{array}$ & $\begin{array}{c}\text { CCR5 } \\
\text { perivascular }\end{array}$ & $\begin{array}{c}\text { CXCR1 } \\
\text { perivascular }\end{array}$ & $\begin{array}{l}\text { DARC } \\
\text { capill. }\end{array}$ & $\begin{array}{l}\text { DARC } \\
\text { middle }\end{array}$ & $\begin{array}{c}\text { CCR5 } \\
\text { int. }\end{array}$ & $\begin{array}{c}\text { CXCR1 } \\
\text { int. }\end{array}$ & Infection \\
\hline АзВ3 & 0.4 & 0.2 & 0.2 & 2 & 2 & 3 & 1 & Yes \\
\hline АзB2 & 0.2 & 0.2 & 0.0 & 1 & 2 & 1 & 0 & No \\
\hline АзB2 & 0.5 & 0.3 & 0.0 & 1 & 2 & 1 & 3 & Yes \\
\hline АзВ2 & 0.4 & 0.1 & 0.2 & 1 & 1 & 1 & 1 & Yes \\
\hline A3BX & 0.6 & 0.4 & 0.1 & 3 & 2 & 2 & 1 & Yes \\
\hline $\mathbf{A 3}^{\#}$ & $0.4 \pm 0.05^{\star \star \star}$ & $0.2 \pm 0.05^{* *}$ & $0.08 \pm 0.03^{*}$ & $1.6 \pm 0.3$ & $2.0 \pm 0.2$ & $1.3 \pm 0.36^{*}$ & $1.3 \pm 0.4$ & \\
\hline A2B3 & 0.4 & 0.3 & 0.0 & 1 & 2 & 1 & 1 & Yes \\
\hline A2B2 & 0.6 & 0.2 & 0.0 & 2 & 2 & 1 & 1 & Yes \\
\hline A2B2 & 0.6 & 0.5 & 0.3 & 1 & 2 & 2 & 2 & Yes \\
\hline A2B2 & 0.7 & 0.4 & 0.0 & 1 & 3 & 1 & 2 & Yes \\
\hline A2B1 & 0.4 & 0.5 & 0.0 & 1 & 3 & 1 & 0 & Yes \\
\hline A2B1 & 0.7 & 0.3 & 0.0 & 1 & 3 & 1 & 1 & Yes \\
\hline A2B1 & ND & 0.0 & 0.0 & 0 & 1 & 1 & 1 & No \\
\hline A2B1 & 0.4 & 0.0 & 0.0 & 1 & 2 & 0 & 0 & No \\
\hline A2B1 & 0.6 & 0.5 & 0.2 & 3 & 3 & 2 & 0 & No \\
\hline A2B0 & 0.5 & 0.3 & 0.3 & 1 & 2 & 0 & 0 & Yes \\
\hline A2BX & 0.4 & 0.5 & 0.1 & 3 & 2 & 2 & 1 & Yes \\
\hline A2BX & 0.6 & 0.1 & 0.0 & 0 & 2 & 0 & 1 & Yes \\
\hline A2BX & 0.4 & 0.4 & 0.4 & 2 & 2 & 2 & 1 & Yes \\
\hline A2BX & 0.7 & 0.1 & 0.1 & 1 & 3 & 1 & 1 & Yes \\
\hline A2 & $0.5 \pm 0.03^{\star \star \star}$ & $0.3 \pm 0.03^{* \star *}$ & $0.08 \pm 0.03^{* *}$ & $1.3 \pm 0.2$ & $2.0 \pm 0.15^{\star *}$ & $1.2 \pm 0.16^{\star \star}$ & $0.9 \pm 0.14$ & \\
\hline
\end{tabular}

also noted in 24 out of 31 biopsies graded A2/A3 and 11 out of 23 graded A0. Haemosiderosis was seen in six out of 24 A2/A3 graded biopsies and in three of the A0 graded biopsies.

\section{Upregulation of DARC expression on capillary vessels in allograft rejection and inflammation}

The control group consisted of 23 patients without signs of acute allograft rejection according to the ISHLT criteria. In all examined samples a small number (median (range) 4 (3-7) per biopsy) of medium-sized to large pre-/post-capillary blood vessels (mostly veins) of the alveolar parenchyma exhibited a distinct DARC signal on endothelial cells (fig. 1a). DARC immunostaining was also readily detectable on vessels of the bronchiolar region. This corresponds to the constitutive pattern described in normal adult lung tissue [15].
Specimens with histological evidence of acute cellular rejection (perivascular lymphocytic infiltrates with/without spilling over into the interstitium) demonstrated an overall increase in the DARC staining of small blood vessels, in particular of capillaries of the alveolar wall (figs $1 \mathrm{c}$ and 2a). This was reflected in the number of foci (two or three per biopsy). At the same time, we noted a stronger expression of DARC on the endothelium of medium-sized blood vessels (tables 1 and 2 and fig. 2b).

Focally restricted expression of the Duffy antigen was also detected in smaller vessels and capillaries of the alveolar septa in 15 out of 23 biopsies without evidence of acute allograft rejection. In the majority of these samples (11 out of 15) concomitant parenchymal inflammation could be observed. The degree, the distribution and the composition of the 


\begin{tabular}{|c|c|c|c|c|c|c|c|c|}
\hline Rejection & \multicolumn{3}{|c|}{ Fraction of positive cells } & \multicolumn{5}{|c|}{ Score } \\
\hline A0B1 & 0 & 0 & 0 & 1 & 2 & 0 & 1 & Yes \\
\hline A0B1 & 0 & 0 & 0 & 1 & 1 & 1 & 1 & Yes \\
\hline A0B1 & 0 & 0 & 0 & 0 & 1 & 0 & 1 & No \\
\hline A0B1 & 0 & 0 & 0 & 0 & 1 & 0 & 0 & No \\
\hline A0B1 & 0 & 0 & 0 & 1 & 2 & 1 & 2 & Yes \\
\hline A0B1 & 0 & 0 & 0 & 0 & 1 & 0 & 1 & No \\
\hline A0B1 & 0 & 0 & 0 & 1 & 2 & 1 & 2 & Yes \\
\hline АОВO & 0 & 0 & 0 & 2 & 2 & 1 & 0 & No \\
\hline АОВО & 0 & 0 & 0 & 2 & 2 & 1 & 2 & Yes \\
\hline АОВO & 0 & 0 & 0 & 0 & 1 & 0 & 1 & No \\
\hline А०BO & 0 & 0 & 0 & 0 & 1 & 0 & 1 & No \\
\hline АОВо & 0 & 0 & 0 & 1 & 2 & 1 & 1 & No \\
\hline АОВО & 0 & 0 & 0 & 1 & 2 & 0 & 1 & No \\
\hline AOBX & 0 & 0 & 0 & 3 & 1 & 1 & 1 & Yes \\
\hline AOBX & 0 & 0 & 0 & 0 & 1 & 0 & 0 & No \\
\hline AOBX & 0 & 0 & 0 & 1 & 1 & 0 & 1 & No \\
\hline AOBX & 0 & 0 & 0 & 1 & 2 & 0 & 1 & Yes \\
\hline AOBX & 0 & 0 & 0 & 1 & 1 & 1 & 2 & Yes \\
\hline
\end{tabular}

Data are presented as mean \pm SEM, unless otherwise stated. Capill.: capillaries; middle: mid-sized vessels; int.: interstitial. ${ }^{\#}: \mathrm{n}=23$.

inflammatory reaction ranged from acute bronchitis to pneumonia with or without signs of organisation. This pattern of DARC expression in infection-mediated lung injury reflects the patchy alveolar staining described in suppurative pneumonia [15].

Out of 15 samples with DARC expression with rejection grade A0, four did not display any signs of infection (histology confirmed by clinical data), but still exhibited foci of DARC antigen-expressing capillaries. A distinguishing feature of the morphology of lung parenchyma of three of them was focal or diffuse haemosiderosis (fig. 1b). An investigation into the case history of these three patients revealed a previous episode of acute rejection ( 3 weeks to 6 months prior to actual biopsy).

When comparing the mean scores of DARC positivity of capillaries in specimens without (A0) and with rejection (A2) A3) and correlating them with the presence of signs of inflammation (most likely caused by infection), we noted that although the increase in the number of DARC-positive capillaries in rejection is notable it is even more significant when infection is added to rejection (fig. 2a). Inflammation alone increased the DARC score in a more dramatic way than mild or moderate vascular rejection. This is even more evident when the DARC score of small to medium-sized vessels is examined. We found the highest DARC scores in samples with both rejection and inflammation and the difference between rejection alone and rejection plus infection is even more striking (fig. 2b). Therefore, both types of injury to the allograft contribute to the increase in DARC-positive vessels.

\section{Interstitial CCR5-positive T-cells are associated with an increased number of DARC-positive capillaries in acute lung rejection}

CCR5-positive cells formed a significant part of the infiltrating cells in the majority of biopsies with histological evidence of acute rejection. Many of them (22 out of 24) were clustered in areas of the alveolar walls, where DARC-expressing capillaries could also be observed on consecutive sections (fig. 1d). In contrast, only six out of 23 biopsies without evidence of acute cellular rejection showed a correlation between DARC-positive capillaries and interstitial CCR5-positive leukocytes (presence of interstitial CCR5-positive cells in at least one area with prominent DARC-positive capillaries). In those samples, either inflammation or haemosiderosis was detected.

\section{Interstitial CXCR1-positive leukocytes are associated with increased numbers of DARC-positive capillaries in lung biopsies with evidence of inflammation}

24 out of 31 specimens with grade A2 and A3 rejection and 11 out of 23 biopsies without histological evidence of rejection 

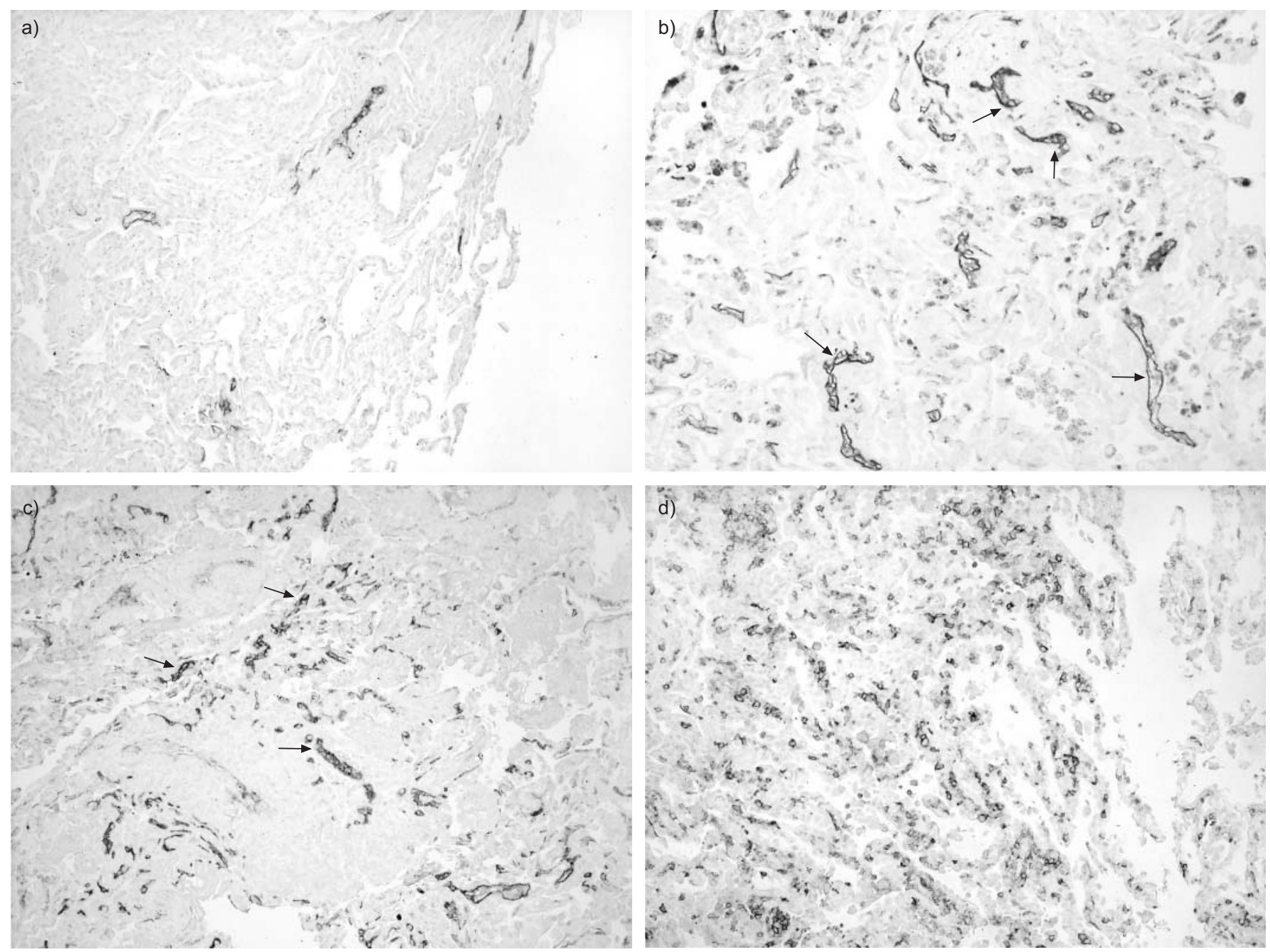

FIGURE 1. Immunohistochemical detection of Duffy antigen/receptor for chemokines (DARC) in parenchymal capillary vessels and of interstitial CCR5-positive cells of lung allografts. Immunohistochemistry of transbronchial lung biopsies stained with monoclonal antibodies against a-c) DARC and d) CCR5. a) Normal parenchyma of transplanted lung with no evidence of rejection or infection shows moderate DARC labelling of endothelial cells of medium-sized (pre-/post-capillary) blood vessels, but absent staining of capillaries of the alveolar septa. b) Transbronchial biopsy negative for acute rejection with features of infection. Distinct DARC labelling of alveolar capillaries and blood vessels of different sizes can be observed (arrows) and the alveolar spaces are filled with haemosiderin-laden macrophages. c) Focus with many DARC-positive capillaries and small blood vessels (arrows) in a transbronchial biopsy from a patient with A3 acute rejection. d) In a parallel section of the same biopsy, CCR5-positive interstitial leukocytes co-localised with DARC-positive capillaries. Magnification $\times 400$.

presented with signs of infection. CXCR1-positive granulocytes, the hallmark of acute inflammation, were present in all specimens with acute bronchitis. Distinct focal infiltration of alveolar parenchyma by CXCR1-positive granulocytes could be seen in different stages of infection-triggered lung injury, as well as in incipient/resolving pneumonia (11 out of 54 patients). In these biopsies (six with rejection grade $\mathrm{A} 2+\mathrm{A} 3$ and five with rejection grade $\mathrm{A} 0$ ) the presence of interstitial CXCR1-expressing leukocytes was observed in at least one area with prominent DARC-positive capillaries per biopsy.

\section{Perivascular mononuclear cells express CXCR3, CCR5 and CXCR1 in acute lung rejection}

Cuffing of blood vessels by activated, CD3-positive T-cells is the hallmark of acute pulmonary rejection. We routinely stained biopsies with antibodies against CD3 in order to assess (semiquantitatively) the density of lymphocytes in the infiltrate and grade acute rejection. This is particularly helpful in biopsies containing only a few blood vessels. In higher degrees of rejection the presence of macrophages, neutrophils and eosinophils within the perivascular infiltrate is more pronounced $(\mathrm{A} 3>\mathrm{A} 2>\mathrm{A} 1)$.

CD3 staining correlated with the amount of perivascular lymphocytes seen by light microscopy: $54.7 \%$ and $38.3 \%$ of all perivascular cells (median percentage) expressed CD3 in A2 and $\mathrm{A} 3$ acute rejection, respectively. A0 rejection is defined by a lack of perivascular lymphocytes; in these areas no CD3expressing cells can be observed. In the studied biopsies, Tcells in the infiltrate strongly expressed CXCR3: $44.2 \%$ of all perivascular cells were CXCR3 positive in $\mathrm{A} 3$ and $50.2 \%$ were positive in A2 acute rejection (median percentage).

The number of CCR5 expressing mononuclear cells in the neighbourhood of blood vessels was considerably smaller: 

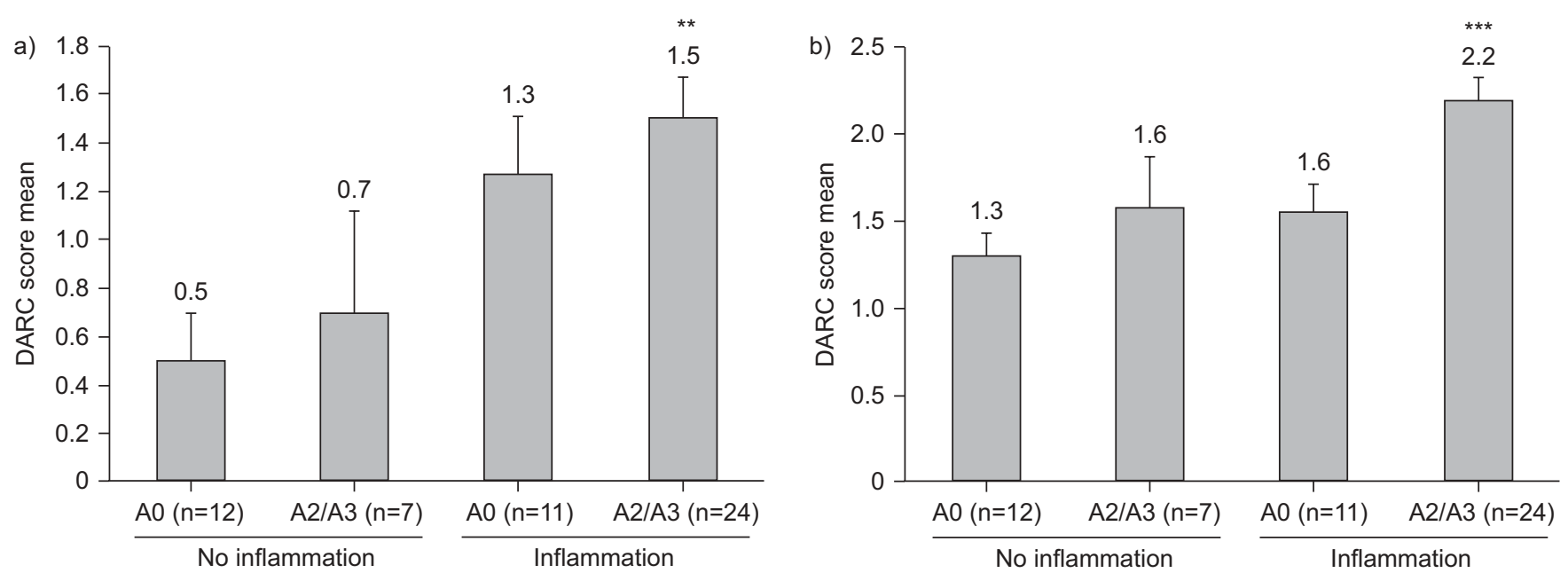

FIGURE 2. Mean Duffy antigen/receptor for chemokines (DARC) scores of a) capillaries and b) medium-sized vessels according to the presence of acute allograft rejection (AO versus $\mathrm{A} 2 / 3$ ) and the presence of inflammation (no inflammation versus inflammation). The error bars illustrate $\mathrm{SEM}$. **: $p<0.01, A 2 / 3$ inflammation versus $A 0$ no inflammation; ${ }^{* \star}: \mathrm{p}<0.001, \mathrm{~A} 2 / 3$ inflammation versus $\mathrm{A0}$ no inflammation).

21.4\% of CCR5-positive cells were observed in infiltrates of A3 acute rejection and $27.9 \%$ in $\mathrm{A} 2$ acute rejection (median percentages) (fig. 3e). CXCR1 expressing perivascular neutrophils demonstrated the highest variability in numbers. Their mean percentage in perivascular infiltrates in $\mathrm{A} 3$ and $\mathrm{A} 2$ rejections varied between 0 and $40 \%$ with a mean of $8.1 \%$ in $\mathrm{A} 2$ and $7.1 \%$ in $\mathrm{A} 3$.

\section{Bronchial/bronchiolar infiltrates contain CXCR3- and CXCR1-positive leukocytes}

Bronchial/bronchiolar infiltrates contained a mixed population of lymphocytes and macrophages in varying density representing the airway part of acute rejection. The density of these infiltrates is reflected in the grading. The immunostaining profile seen in B1 and B2 acute bronchial/bronchiolar rejection
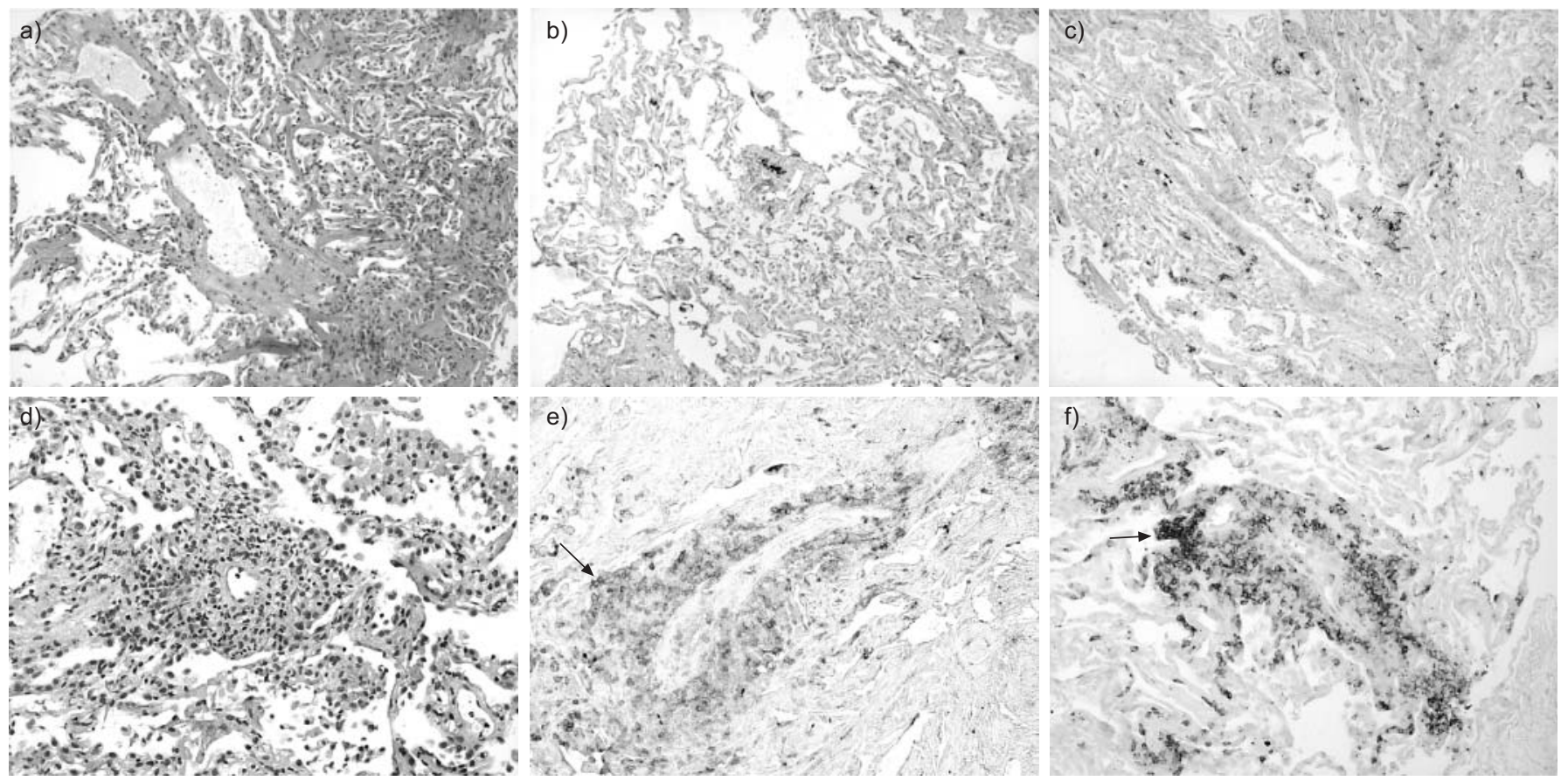

FIGURE 3. Distribution of CXCR3- and CCR5-positive cells in lung allografts. Transbronchial biopsies of lung allografts are illustrated in a-C) a central medium-sized blood vessel with no evidence of perivascular infiltrate corresponding to A0 rejection and $\mathrm{d}-\mathrm{f}$ ) a dense perivascular infiltrate with expansion of the adventitial space, but no involvement of alveolar walls consistent with grade A2 rejection. a, d) Haematoxylin and eosin staining. b, e) Immunohistochemistry for CCR5-positive mononuclear cells. b) No perivascular CCR5-positive cells were observed. e) The CCR5-positive fraction of the perivascular infiltrate (arrow) mainly shows large cells with a weak to moderate membrane staining. c, f) Immunohistochemistry for CXCR3 expression by lung T-cells. f) Strongly stained CXCR3-positive lymphocytes (arrow) can be demonstrated in the perivascular space in A2 rejection but are absent in vessel walls with no infiltrate (c). Only scant CXCR3-positive small lymphocytes can be seen in the alveolar septa of biopsies negative for acute rejection. Magnification $\times 400$. 
shows cuffing of bronchioles and/or bronchi with intact epithelium by CXCR3-positive leukocytes. Higher grades of bronchial/bronchiolar rejection (in our collective B2-B3) had a varying number of infiltrating CXCR3-positive activated lymphocytes in the epithelium. Additional presence of CXCR1positive neutrophils in the denser band-like infiltrate of the submucosa was also often observed. B2 and B3 grading was ascribed to rejection in cases where infection could be clinically and histomorphologically excluded. Prominence of CXCR1positive neutrophils either in exudate and/or in the airway epithelium was consistent with (bacterial) infection concomitant with acute rejection (fig. $4 a$ and b).

\section{DISCUSSION}

This study includes the largest cohort of human lung allograft biopsies studied for the expression of three chemokine receptors and the chemokine binding protein DARC.

DARC is a seven transmembrane protein that binds a variety of pro-inflammatory CC and CXC chemokines [18, 19]. It was first identified as a red blood group antigen involved in transfusion reactions and was later shown to be a co-receptor for malaria parasites [13, 20-22]. Besides red blood cells, DARC is expressed on endothelial cells, notably on high endothelial cells, the site of lymphocyte recirculation [23].

In normal lung tissue constitutive expression of DARC was described on the endothelium of medium-sized parenchymal and bronchiolar vessels [15]. In acute suppurative pneumonia DARC antigen expression is newly present on alveolar septa. These vascular beds with low-shear stress are thought to be the sites of leukocyte extravasation. This is partially due to an environment favourable for interactions between chemokines and their receptors. An increased number of DARC-positive interstitial peritubular capillaries has been demonstrated in renal allograft rejection $[24,25]$. In the kidney, antibody and cell-mediated rejection both resulted in an increased number of DARC-positive peritubular capillaries [25]. Interestingly, the combination of both forms of allograft injury resulted in an additional effect on the number of DARC-positive peritubular capillaries.

This study provides the first evidence that endothelial expression of DARC is upregulated in acute lung allograft rejection. Samples with no or minimal evidence of rejection presented with a focally restricted and infrequent expression of DARC in smaller blood vessels and capillaries of the alveolar septa. This is consistent with the pattern described in normal parenchyma. In acute mild and moderate lung allograft rejection (A2, A3) we could observed an increase in DARC expression on the endothelium of all sizes of blood vessels, most prominently at the small/capillary level. Additionally, inflammation (most likely related to infection) increased the number of DARC-positive vessels. The combination of rejection and infection resulted in the highest numbers of DARCpositive capillaries, and only this combination resulted in an increased number of medium-sized DARC-positive vessels.

This pattern of DARC-positive capillaries mirrored pattern observed in the renal interstitium in renal allograft rejection, except that it seemed to persist after acute rejection had resolved [24, 25]. In an attempt to keep the morphological entities as stringent as possible, all of the examined biopsies (one per patient) were performed at time points when no suspicion or clinical evidence of chronic airway rejection (obliterative bronchiolitis) was present. Prominent foci of DARC-expressing capillaries could be found in A0 lung samples rich in haemosiderin-carrying macrophages. Although haemosiderosis is considered a nonspecific finding indicating past endothelial injury and blood extravasation, it is nevertheless a characteristic residual feature of rejection episodes in lung allografts. As endothelial DARC is involved both in transcytosis of chemokines into the bloodstream as well as induction of leukocyte emigration, persisting DARC induction in these samples could be linked to a role in restoration of chemokine homeostasis [12]. a)

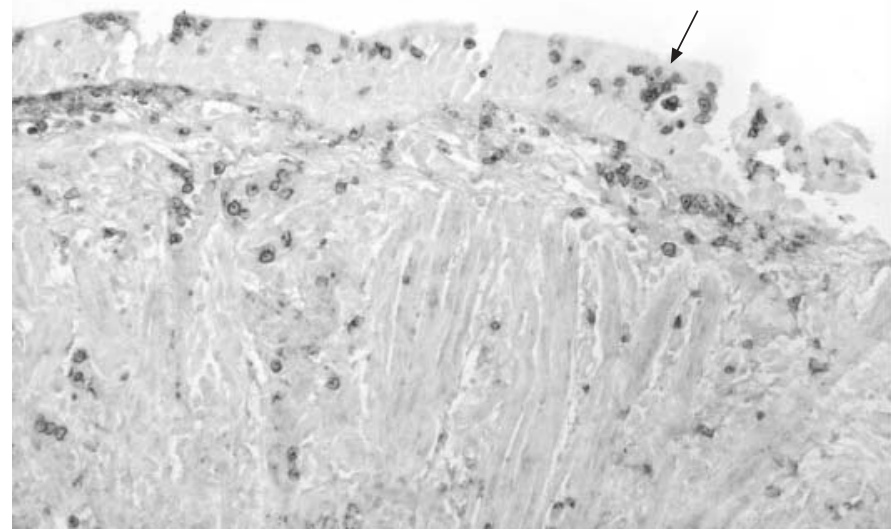

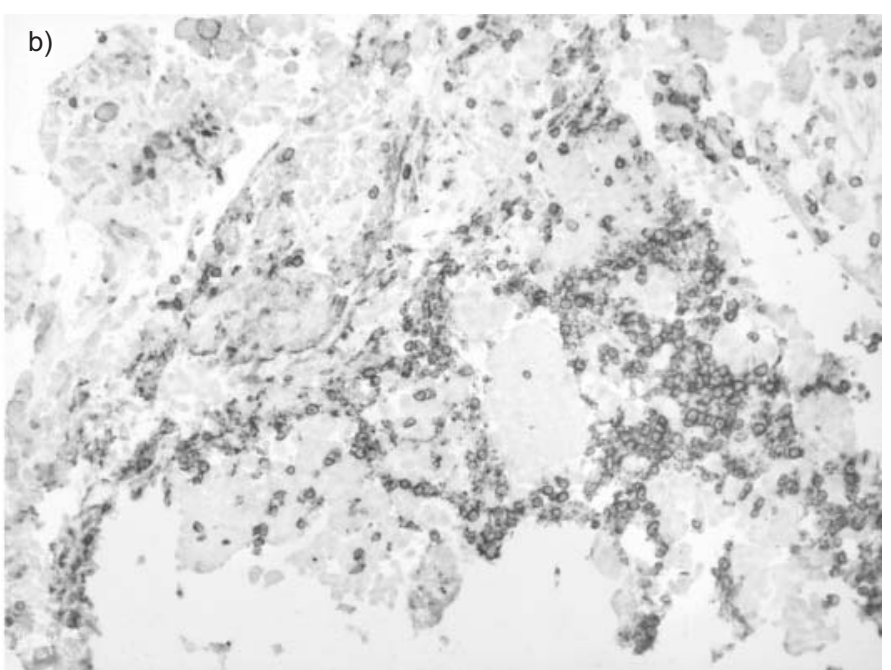

FIGURE 4. CXCR1-expressing leukocytes in lung allografts with evidence of infection. Immunohistochemistry for CXCR1. a) High-power view of a bronchiolar wall with intact columnar epithelium infiltrated by polymorphs expressing CXCR1 (arrow). Magnification $\times 800$. b) Fibrinopurulent exudate containing many CXCR1-expressing polymorphs. Magnification $\times 200$ 
Upregulation of DARC in episodes of mild or moderate lung allograft rejection was associated with prominent recruitment of CCR5-positive cells at the site of rejection and in the interstitium. Previous studies have revealed an upregulation of CCL5/RANTES in solid organ allografts. A significant increase in CCL5/RANTES protein was demonstrated in bronchoalveolar lavages from patients with lung rejection with or without concomittant cytomegalovirus (CMV) infection [2628]. These studies also demonstrated that the pulmonary CMV-associated increase in bronchoalveolar lavage fluid CC chemokines can predict the development of obliterative bronchiolitis. In rat lung allografts the expression of CCL5/ RANTES was associated with recruitment of cells expressing CCR1 and CCR5, and treatment with antibodies against CCL5/RANTES significantly reduced inflammatory cell influx [10]. The functional role of DARC in vascularised allografts is still under intense investigation. A differential effect is postulated for DARC on red blood cells (most likely scavenging chemokines), whereas endothelial DARC might be involved in the transendothelial transport and apical presentation [12]. Therefore, it can be hypothesised that with the increased number of DARC-positive vessels (in acute rejection with and without infection) the pro-inflammatory role of DARC might become more important. This would explain the accumulation of CCR5-positive cells in these areas.

CXCR3, the shared receptor of the chemoattractants CXCL9/ Mig, CXCL10/IP-10 and CXCL11/I-TAC (interferon-inducible T-cell $\alpha$ chemoattractant), is a prominent mediator of cytotoxic T-lymphocyte responses not only in allograft rejection but also in other Th1-related disorders. In our population, episodes of grade A2 or greater lung allograft rejection were associated with striking expression of CXCR3 and lesser expression of CCR5 chemokine receptors by perivascular lymphocytes. These findings extend the results of a previous study where immunohistochemical analysis of selected cases of human lung allograft rejection demonstrated CXCR3-positive T-cell infiltrates in perivascular and interstitial areas [29].

In the present study we observed recruitment of CXCR1-positive neutrophils both in severe forms of lung allograft rejection and in acute (bacterial) infection. Activated neutrophils have been implicated in the pathogenesis of tissue injury in early and late events of lung transplantation. They are sequestered after reperfusion, or with episodes of infection and/or rejection, and activated by CXCL8/IL-8 and its two receptors CXCR1 and CXCR2 [6, 30]. Several lines of evidence suggested that neutrophils could play a key role in the development of acute cellular graft rejection through direct or indirect mechanisms, such as Th1-specific polarisation of T-lymphocytes [31]. In our study population, CXCR1-positive neutrophils were seen at sites of higher grades of acute rejection (A2-3 and A3), where these are components of the perivascular infiltrates. We also observed a varying number of CXCR1-positive neutrophils in segments of the bronchial tree. Neutrophils infiltrating the mucosa and/or submucosa were either a feature of higher grades of acute bronchial/bronchiolar rejection (B2, B3) or evidence of airway inflammation. A clear distinction of the two events on morphological grounds alone was only possible when CXCR1-positive neutrophils were seen to cluster and form purulent exsudates. Either cause of bronchial/bronchiolar infiltration is known to create a pro-inflammatory environment that predisposes to obliterative bronchiolitis, the most important long-term complication after lung transplantation [32, 33].

In summary, this is the first study that demonstrates a prominent induction of the chemokine binding protein DARC at the site of CCR5-positive T-cell infiltration in human lung allografts. It was associated with recruitment of CXCR1positive neutrophils not only in acute infection but also in severe forms of lung allograft rejection. CXCR3 was the receptor most commonly expressed by lymphocytes during lung allograft rejection. The results of this study suggest that endothelial DARC and chemokine receptors on leukocytes, together with their major ligands CCL5/RANTES and CXCL8/ IL-8, may enhance recruitment to sites of rejection and promote cell-mediated lung injury. The studied chemokine receptors could be viewed as potential therapeutic targets and further studies need to address the potential of antagonists (single and in combination) in lung allograft models.

\section{SUPPORT STATEMENT}

S. Segerer is supported by the Hartmann Müller Stiftung Foundation (Zurich, Switzerland).

\section{STATEMENT OF INTEREST}

None declared.

\section{REFERENCES}

1 Lynch JP 3rd, Saggar R, Weigt SS, et al. Overview of lung transplantation and criteria for selection of candidates. Semin Respir Crit Care Med 2006; 27: 441-469.

2 Snyder LD, Palmer SM. Immune mechanisms of lung allograft rejection. Semin Respir Crit Care Med 2006; 27: 534-543.

3 Murphy PM, Baggiolini M, Charo IF, et al. International union of pharmacology. XXII. Nomenclature for chemokine receptors. Pharmacol Rev 2000; 52: 145-176.

4 Hancock WW. Chemokines and transplant immunobiology. J Am Soc Nephrol 2002; 13: 821-824.

5 Colvin BL, Thomson AW. Chemokines, their receptors, and transplant outcome. Transplantation 2002; 74: 149-155.

6 De Perrot M, Sekine Y, Fischer S, et al. Interleukin-8 release during early reperfusion predicts graft function in human lung transplantation. Am J Respir Crit Care Med 2002; 165: 211-215.

7 Belperio JA, Keane MP, Burdick MD, et al. CXCR2/CXCR2 ligand biology during lung transplant ischemia-reperfusion injury. J Immunol 2005; 175: 6931-6939.

8 Belperio JA, Keane MP, Burdick MD, et al. Role of CXCL9/CXCR3 chemokine biology during pathogenesis of acute lung allograft rejection. J Immunol 2003; 171: 4844-4852.

9 Sekine Y, Yasufuku K, Heidler KM, et al. Monocyte chemoattractant protein-1 and RANTES are chemotactic for graft infiltrating lymphocytes during acute lung allograft rejection. Am J Respir Cell Mol Biol 2000; 23: 719-726.

10 Belperio JA, Burdick MD, Keane MP, et al. The role of the CC chemokine, RANTES, in acute lung allograft rejection. J Immunol 2000; 165: 461-472.

11 Nibbs R, Graham G, Rot A. Chemokines on the move: control by the chemokine "interceptors" Duffy blood group antigen and D6. Semin Immunol 2003; 15: 287-294.

12 Pruenster M, Mudde L, Bombosi $\mathrm{P}$, et al. The Duffy antigen receptor for chemokines transports chemokines and supports their promigratory activity. Nat Immunol 2009; 10: 101-108.

13 Hadley TJ, Peiper SC. From malaria to chemokine receptor: the emerging physiologic role of the Duffy blood group antigen. Blood 1997; 89: 3077-3091. 
14 Rot A, von Andrian UH. Chemokines in innate and adaptive host defense: basic chemokinese grammar for immune cells. Annu Rev Immunol 2004; 22: 891-928.

15 Lee JS, Frevert CW, Thorning DR, et al. Enhanced expression of Duffy antigen in the lungs during suppurative pneumonia. J Histochem Cytochem 2003; 51: 159-166.

16 Stewart S, Fishbein MC, Snell GI, et al. Revision of the 1996 working formulation for the standardization of nomenclature in the diagnosis of lung rejection. J Heart Lung Transplant 2007; 26: 1229-1242.

17 Bruhl H, Vielhauer V, Weiss M, et al. Expression of DARC, CXCR3 and CCR5 in giant cell arteritis. Rheumatology (Oxford) 2005; 44: 309-313.

18 Gardner L, Patterson AM, Ashton BA, et al. The human Duffy antigen binds selected inflammatory but not homeostatic chemokines. Biochem Biophys Res Commun 2004; 321: 306-312.

19 Horuk R, Wang ZX, Peiper SC, et al. Identification and characterization of a promiscuous chemokine-binding protein in a human erythroleukemic cell line. J Biol Chem 1994; 269: 1773017733.

20 Middleton J, Patterson AM, Gardner L, et al. Leukocyte extravasation: chemokine transport and presentation by the endothelium. Blood 2002; 100: 3853-3860.

21 Cartron JP, Bailly P, Le Van Kim C, et al. Insights into the structure and function of membrane polypeptides carrying blood group antigens. Vox Sang 1998; 74: Suppl. 2, 29-64.

22 Darbonne WC, Rice GC, Mohler MA, et al. Red blood cells are a sink for interleukin 8, a leukocyte chemotaxin. J Clin Invest 1991; 88: 1362-1369.

23 Girard JP, Baekkevold ES, Yamanaka T, et al. Heterogeneity of endothelial cells: the specialized phenotype of human high endothelial venules characterized by suppression subtractive hybridization. Am J Pathol 1999; 155: 2043-2055.
24 Segerer S, Regele H, Mac KM, et al. The Duffy antigen receptor for chemokines is up-regulated during acute renal transplant rejection and crescentic glomerulonephritis. Kidney Int 2000; 58: 1546-1556.

25 Segerer S, Bohmig GA, Exner M, et al. When renal allografts turn DARC. Transplantation 2003; 75: 1030-1034.

26 Monti G, Magnan A, Fattal M, et al. Intrapulmonary production of RANTES during rejection and CMV pneumonitis after lung transplantation. Transplantation 1996; 61: 1757-1762.

27 Reynaud-Gaubert M, Marin V, Thirion X, et al. Upregulation of chemokines in bronchoalveolar lavage fluid as a predictive marker of post-transplant airway obliteration. J Heart Lung Transplant 2002; 21: 721-730.

28 Weigt SS, Elashoff RM, Keane MP, et al. Altered levels of CC chemokines during pulmonary CMV predict BOS and mortality post-lung transplantation. Am J Transplant 2008; 8: 1512-1522.

29 Agostini C, Calabrese F, Rea F, et al. CXCR3 and its ligand CXCL10 are expressed by inflammatory cells infiltrating lung allografts and mediate chemotaxis of T cells at sites of rejection. Am J Pathol 2001; 158: 1703-1711.

30 Meyer KC, Nunley DR, Dauber JH, et al. Neutrophils, unopposed neutrophil elastase, and $\alpha 1$-antiprotease defenses following human lung transplantation. Am J Respir Crit Care Med 2001; 164: 97-102.

31 Buonocore S, Surquin M, Le Moine A, et al. Amplification of T-cell responses by neutrophils: relevance to allograft immunity. Immunol Lett 2004; 94: 163-166.

32 Elssner A, Vogelmeier C. The role of neutrophils in the pathogenesis of obliterative bronchiolitis after lung transplantation. Transpl Infect Dis 2001; 3: 168-176.

33 Bharat A, Narayanan K, Street T, et al. Early posttransplant inflammation promotes the development of alloimmunity and chronic human lung allograft rejection. Transplantation 2007; 83: 150-158. 